\title{
Amplification of near-infrared fluorescence in semiconducting polymer nanoprobe for grasping the behaviors of systemically administered endothelial cells in ischemia treatment
}

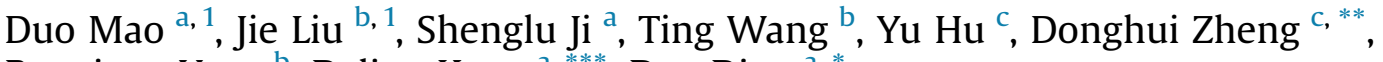

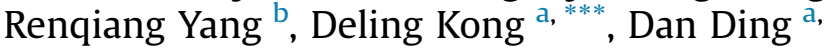 \\ a State Key Laboratory of Medicinal Chemical Biology, Key Laboratory of Bioactive Materials, Ministry of Education, and College of Life Sciences, Nankai \\ University, Tianjin 300071, China \\ ${ }^{\mathrm{b}}$ CAS Key Laboratory of Bio-based Materials, Qingdao Institute of Bioenergy and Bioprocess Technology, Chinese Academy of Sciences, Qingdao 266101, \\ China \\ ${ }^{c}$ Department of Nephrology, Huai'an Hospital Affiliated to Xuzhou Medical College and Huai'an Second Hospital, Huai'an 223002, China
}

\section{A R T I C L E I N F O}

Article history:

Received 14 February 2017

Received in revised form

12 July 2017

Accepted 30 July 2017

Available online 2 August 2017

Keywords:

Semiconducting $\pi$-conjugated polymer Fluorescence resonance energy transfer Endothelial cell tracking

Critical limb ischemia

\begin{abstract}
A B S T R A C T
To date, there have been few studies on using fluorescent cell trackers for non-invasively monitoring the in vivo fate of systemically administered cells. This is because only a relatively small number of cells can reach the disease site post systemic infusion, and thus achieving ideal in vivo cell tracking requires that the fluorescent cell trackers should hold combined merits of ultrahigh near-infrared (NIR) fluorescence, negligible interference on cell behavior and function, excellent retention within cells, as well as accurate long-term cell tracking ability. To address this challenge, we herein developed a highly NIR fluorescent nanoprobe (SPN) based on semiconducting $\pi$-conjugated polymers (SPs), by synthesis of a NIR SPemitter, employment of fluorescence resonance energy transfer (FRET) strategy, and optimization of different FRET donor SPs. Due to the 53.7-fold intra-particle amplification of NIR fluorescence, the SPN could track as few as 2000 endothelial cells (ECs) upon intra-arterial injection into critical limb ischemia (CLI)-bearing mice, showing much higher sensitivity in ECs tracking compared with the most popular commercial cell trackers. What's more, the SPN could provide precise information on the behaviors of systemically injected ECs in CLI treatment including the in vivo fate and regenerative contribution of ECs for at least 21 days.
\end{abstract}

(c) 2017 Published by Elsevier Ltd.

\section{Introduction}

Ischemia, a restriction in blood supply to tissues, often causes tissue damage or dysfunction [1]. Among many an ischemic disease such as myocardial infarction, stroke, critical limb ischemia (CLI), and so on [2-4], CLI is an advanced stage of peripheral artery disease that is highly associated with diabetes mellitus. The patients suffering diabetes are faced with a heavy risk of major amputation caused by CLI $[5,6]$. Thereby, a great amount of research and clinical

\footnotetext{
* Corresponding author.

** Corresponding author.

*** Corresponding author.

E-mail addresses: zddwjj@126.com (D. Zheng), kongdeling@nankai.edu.cn (D. Kong), dingd@nankai.edu.cn (D. Ding).

1 These authors have contributed equally.
}

efforts have focused on cell-based therapy strategies (e.g., stem cells, endothelial progenitor cells, or endothelial cells) for CLI treatment, showing the significant outcome in improving limb perfusion [7-9]. With the flourishing progress of cell therapy, effective exogenous cell trackers that can report the in vivo fate of administrated cells in an accurate and long-term manner are in urgent pursuit. Although a variety of imaging contrast agents have been developed as cell trackers, rather limited studies can clearly prove that whether their agents still precisely label the original cells in vivo over an exceedingly long period of time and how the cells dedicate to the disease therapy by virtue of the agent labeling [10].

To achieve ideal long-term cell tracking, a sensitive imaging modality with high resolution in combination with a biostable and biocompatible contrast agent are required. Compared with versatile imaging techniques, fluorescence imaging holds the advantages 
of excellent sensitivity, high resolution at cellular level, maneuverable instruments, and good safety [11]. In particular, bioimaging in the near-infrared (NIR) window (650-900 nm) offers the merits of low interferential autofluorescence and high tissue permeability [12-14]. In comparison to traditional fluorescent materials, semiconducting $\pi$-conjugated polymers (SPs) have been well accepted as a class of advanced fluorescent materials with high extinction coefficient, bright emission, large photobleaching threshold, and good biocompatibility [15-19]. Recently, SP-based nanoparticles (SPNs) have received considerable attention as they integrate both the advantages of SPs and nanotechnology [20-42]. As compared to inorganic quantum dots (QDs), SPNs have been reported to show higher fluorescence by tens of times, better biological stability, and much lower cytotoxicity [43-45], which make them very promising as next generation of fluorescent nanodots.

In clinical cell therapy, systemic infusion including intravenous (i.v.) and intra-arterial (i.a.) injections is the most common method for administration of cells [46]. Therefore, understanding the behaviors of systemically administered cells in disease treatment becomes increasingly important and urgent. Until now, however, there have been few published studies on using fluorescent cell trackers for non-invasively monitoring the in vivo fate of systemically administered cells. This is because only a relatively small number of cells can reach the disease site post systemic infusion, and thus achieving ideal in vivo cell tracking requires that the fluorescent cell trackers should hold combined merits of ultrahigh NIR fluorescence, superb biological and photophysical stabilities, negligible interference on cell behavior and function, excellent retention within cells, as well as precise long-term cell tracking ability [47-50]. This longstanding challenge motivated us to explore advanced fluorescent cell trackers to meet all the aforementioned requirements.

In this contribution, we reported the development of a highly NIR fluorescent SPN for close monitoring of in vivo behaviors of systemically administered human umbilical vein endothelial cells (ECs) in the treatment of CLI. A NIR SP-emitter, PFDBD10 (Fig. 1A), was synthesized and used as the fluorescence resonance energy transfer (FRET) acceptor. Two visible-light-harvesting SPs, PFBD and PFBT (Fig. 1A), were utilized as the FRET donor candidates. Using a facile nanoengineering method, the donor and acceptor SPS were co-formulated into one nanoparticle to realize FRET, achieving tremendously amplified NIR fluorescence. Importantly, we demonstrated that PFBD could serve as a better FRET donor than PFBT in terms of higher absorptivity at $488 \mathrm{~nm}$, larger enhancement of acceptor emission, and higher photoluminescence (PL) quantum yield (QY) of SPNs in water. After surface functionalization using an EC proteoglycan-binding peptide, the PFBD/PFDBD10-PBP-SPNs were applied to trace systemically administered ECs in a CLIbearing mouse model. By virtue of the ultrahigh NIR brightness, PFBD/PFDBD10-PBP-SPNs could not only accurately and dynamically track the ECs after i.a. or i.v. injection for as long as 3 weeks, but also show much higher sensitivity in tracing of ECs in vivo than the most popular commercial cell trackers, Qtracker ${ }^{\circledR} 655$ and PKH26. Besides, the PFBD/PFDBD10-PBP-SPNs labeling was able to reveal how the systemically administered ECs devote to CLI therapy in vivo, offering important information on EC therapy of ischemia.

\section{Materials and methods}

\subsection{Synthesis of PFDBD10}

A Schlenk tube was charged with compound 1 (192.6 mg, $0.3 \mathrm{mmol}$ ), compound 2 (131.5 $\mathrm{mg}, 0.24 \mathrm{mmol})$, compound $\mathbf{3}$ $(26.5 \mathrm{mg}, 60.0 \mu \mathrm{mol})$, as well as $\mathrm{Pd}\left(\mathrm{PPh}_{3}\right)_{4}(4.6 \mathrm{mg}, 4.0 \mu \mathrm{mol})$ in toluene $(8.0 \mathrm{~mL})$ before it was sealed with a rubber septum. The
Schlenk tube was degassed with three freeze-pump-thaw cycles to remove air. After the mixture was heated to $85{ }^{\circ} \mathrm{C}$, an aqueous $\mathrm{Et}_{4} \mathrm{NOH}$ solution $(20 \mathrm{wt} \%, 2.0 \mathrm{~mL}$ ) was added to initiate the reaction. The mixture was kept at $85^{\circ} \mathrm{C}$ and stirred for $20 \mathrm{~h}$, followed by precipitation from methanol $(100 \mathrm{~mL})$. The precipitate was collected by filtration and dried, and then dissolved in chloroform $(200 \mathrm{~mL})$. The solution was then washed with water for 3 times, and dried over $\mathrm{MgSO}_{4}$. After solvent removal, the residue was poured into methanol $(100 \mathrm{~mL})$ to give polymer as fibres. The polymer was further purified by Soxhlet extraction in acetone for $24 \mathrm{~h}$ to remove the fraction with small molecular weight. The precipitation cycles in toluene/methanol were then repeated twice to afford the final polymer (142.6 mg, 63\%) as bright red fibres. ${ }^{1} \mathrm{H}$ NMR $(600 \mathrm{MHz}$, $\mathrm{CDCl}_{3}, \mathrm{ppm}$ ) $\delta: 8.17$ (br), 7.84 (br), 7.78-7.67 (br), 7.52 (br), 7.37 (br), 2.12 (br), 1.25-1.14 (br), 0.82 (br).

\subsection{Preparation of SPNs}

Predetermined amounts of donor SP (PFBD or PFBT), and/or PFDBD10 (fixed at $0.3 \mathrm{mg}$ ) as well as maleimide-bearing lipid-PEG (fixed at $3 \mathrm{mg}$ ) were dissolved in $1 \mathrm{~mL}$ of tetrahydrofuran (THF), which was dropped into $9 \mathrm{~mL}$ of water under sonication using a microtip probe sonicator at $12 \mathrm{~W}$ output. The mixture was then sonicated for another $60 \mathrm{~s}$. After evaporation of THF, the SPN suspensions were filtered through a $0.45 \mu \mathrm{m}$ syringe driven filter. The amounts of donor SP/PFDBD10 encapsulated into the lipid-PEG matrix were determined from the absorption spectra with reference to a calibration curve of donor SP and PFDBD10 in THF. Generally, the encapsulation efficiency of SP defined as the ratio of the amount of SP encapsulated into the SPNs to the total amount of SP in the feed mixture was $>90 \%$. To conjugate proteoglycanbinding peptides (PBPs) on the SPNs, $0.2 \mu \mathrm{mol}$ peptides were added into the SPN suspension, which was allowed to react for $12 \mathrm{~h}$. The free PBPs without conjugation on SPNs were subsequently removed by ultrafiltration.

\subsection{Cell culture}

Human umbilical vein endothelial cells (ECs) were maintained in EGM-2 (endothelial cell growth medium-2) medium (Lonza, USA) in a $37{ }^{\circ} \mathrm{C}$ incubator under a humidified $95 \%$ air and $5 \% \mathrm{CO}_{2}$ atmosphere. The culture medium was changed twice a week. The ECs of passage 3 were used for all the experiments in this work.

\subsection{In vitro tracking of ECs}

ECs $\left(2 \times 10^{6}\right)$ were seeded in a 6 -well plate and cultured at $37^{\circ} \mathrm{C}$. After removal of the medium and washing with $1 \times$ PBS buffer, the ECs were exposed to $1 \mathrm{nM}$ PFBD/PFDBD10-PBP-SPNs for $4 \mathrm{~h}$ at $37{ }^{\circ} \mathrm{C}$. After that, the ECs were washed twice with $1 \times$ PBS buffer, digested by tripsin-EDTA, and then subcultured for 1,5 and 10 days, respectively. The ECs subcultured for different time points were imaged by confocal laser scanning microscopy (CLSM; Leica TSC SP8, Germany) with excitation at $488 \mathrm{~nm}$ and signal collection above $560 \mathrm{~nm}$ (pinhole: $65.5 \mu \mathrm{m}$ ). The fluorescence intensities of SPN-labeled ECs were then assessed by flow cytometry (Becton Dickinson, San Jose, CA, USA) measurements. Ten thousand events were counted for each sample to plot the histogram (excitation at $488 \mathrm{~nm} ; 680 / 20 \mathrm{~nm}$ bandpass filter). The in vitro ECs tracking study using commercial Qtracker ${ }^{\circledR} 655$ as a control was also performed following the same procedures.

\subsection{Capillary tube formation in vitro}

PFBD/PFDBD10-PBP-SPN-labeled or untreated ECs were 

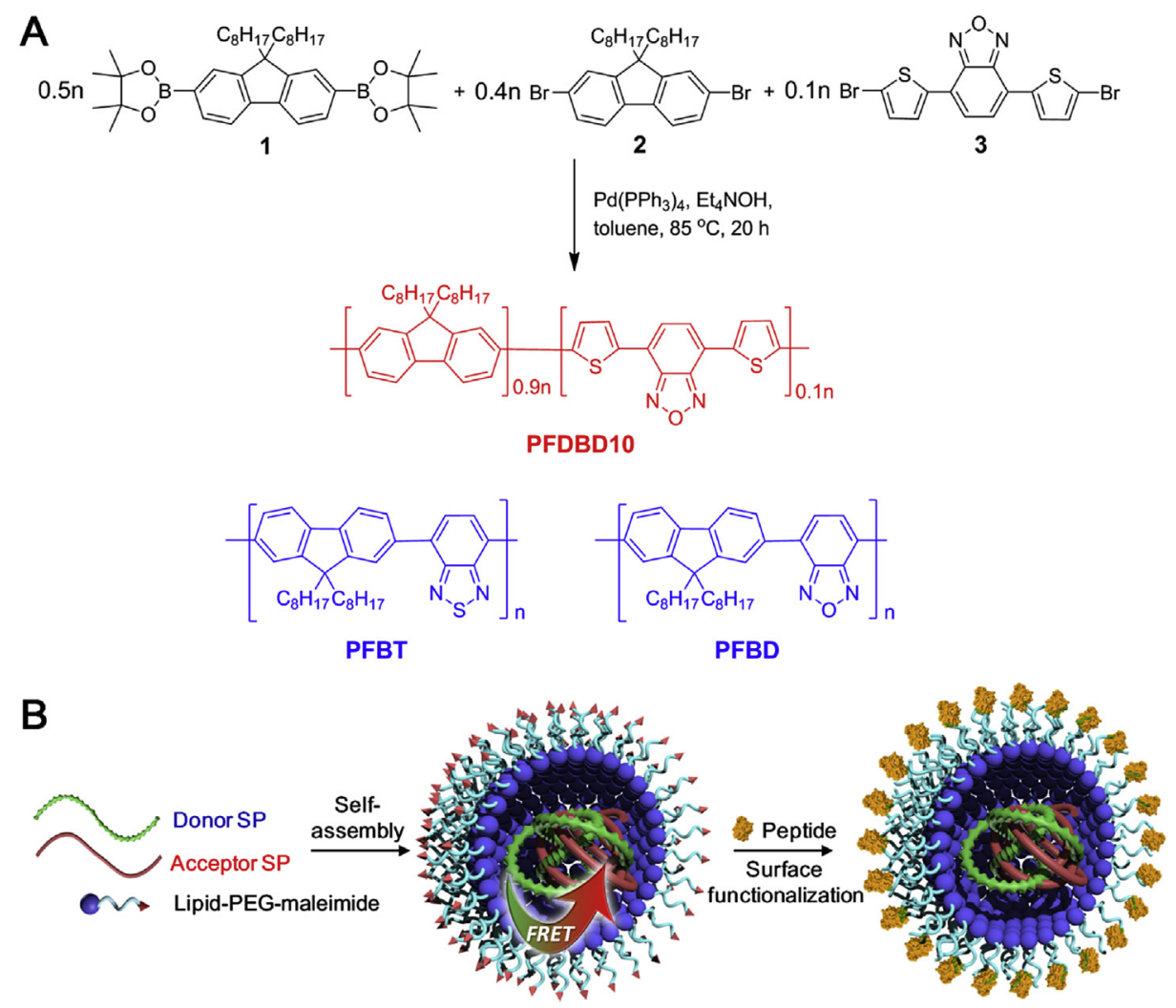

Fig. 1. (A) The synthetic route toward PFDBD10 and chemical structures of PFBT and PFBD. (B) Schematic illustration of the preparation of PFBD/PFDBD10-PBP-SPNs.

harvested through trypsinization and plated in 24-well culture plates $\left(5 \times 10^{4}\right.$ cells/well) pre-coated with $200 \mu \mathrm{L}$ of Matrigel (BD Biosciences) in endothelial basal media (Lonza) supplemented with $1 \%$ fetal bovine serum (FBS). After $24 \mathrm{~h}$, ECs were imaged and captured with CLSM. The tube numbers in 5 random fields $(200 \times)$ from each well were calculated. The results (tube numbers per well) were based on 3 independent experiments.

\subsection{Animals and critical limb ischemia mouse model}

All animal studies were performed in compliance with the guidelines set by Tianjin Committee of Use and Care of Laboratory Animals, and the overall project protocols were approved by the Animal Ethics Committee of Nankai University. 8-10 week-old male $\mathrm{BALB} / \mathrm{c}$ nude mice that were purchased from the Laboratory Animal Center of the Academy of Military Medical Sciences (Beijing, China) were used to establish the critical limb ischemia (CLI) mouse model. Briefly, after anesthetization of the mice, the left femoral artery was ligated via a skin incision with 7-0 silk and cut from just above the deep femoral artery to the popliteal artery.

\subsection{In vivo tracking of $E C S$}

The ECs were labeled with PFBD/PFDBD10-PBP-SPNs (1 nM) as above-mentioned. For non-invasive fluorescence imaging, the CLIbearing mice were randomized into two groups and each group contained 6 mice. $1 \times 10^{6}$ SPN-labeled ECs were injected into each mouse in Group 1 via femoral artery (i.a.) and each mouse in Group 2 via tail vein (i.v.), respectively. The Maestro EX in vivo fluorescence imaging system (CRi, Inc.) was utilized to image the EC-treated mice in two groups at designated time intervals by placing the anesthetized mice on the equipped platform (excitation filter: $435-480 \mathrm{~nm}$; signal collection from 500 to $900 \mathrm{~nm}$ in $10 \mathrm{~nm}$ steps; exposure time: $200 \mathrm{~ms}$ ). Maestro software was used to remove the mouse autofluorescence. For ex vivo tissue imaging, the mice i.a. and i.v. injected with SPN-labeled ECs, respectively, were sacrificed at each time point ( $\mathrm{n}=3$ mice for each group and each time point), and then the ischemic and contralateral hind limbs as well as a variety of normal organs were isolated for ex vivo imaging using the Maestro system.

\subsection{Statistical analysis}

Quantitative data were expressed as mean \pm standard deviation. Statistical comparisons were made by ANOVA analysis and twosample Student's $t$-test. $P$ value $<0.05$ was considered statistically significant.

\section{Results and discussion}

\subsection{Synthesis and characterization of PFDBD10 and SPNs}

Fig. 1A illustrated the synthetic route toward PFDBD10. PFDBD10 was synthesized in 63\% yield via palladium-catalyzed Suzuki polymerization from the monomers: 2,7-bis(4,4,5,5-tetramethyl1,3,2-dioxaborolan-2-yl)-9,9-dioctylfluorene 1, 2,7-dibromo-9,9dioctylfluorene $\mathbf{2}$, and 4,7-bis(5-bromothiophen-2-yl)-2,1,3benzoxadiazole 3 . The final product was then characterized by ${ }^{1} \mathrm{H}$ NMR (Supplementary Fig. S1). Gel permeation chromatography (GPC) result revealed that PFDBD10 possessed a number average molecular weight $\left(M_{\mathrm{n}}\right)$ of 32,165 with a polydispersity index of 2.1. PFDBD10 was easily soluble in common organic solvents such as 
tetrahydrofuran (THF). To render PFDBD10 with water solubility and surface functionalization flexibility, a simple nanoprecipitation method was employed to prepare PFDBD10-doped SPNs (PFDBD10SPNs) utilizing amphiphilic maleimide-bearing lipid-PEG as the doping matrix. The PFDBD10-SPNs possessed hydrophobic lipid/ PFDBD10 entanglements as the core and hydrophilic PEGmaleimide chains as the outer layer to stabilize the nanoparticles (Fig. 1B).

The absorption and PL spectra of PFDBD10-SPNs in water were displayed in Fig. 2A. PFDBD10-SPNs had two absorption maxima at 383 and $540 \mathrm{~nm}$, respectively, and an emission peak centered at $642 \mathrm{~nm}$ with a shoulder at around $700 \mathrm{~nm}$. Achieving effective FRET required the good overlap of the spectra of donor emission and acceptor absorption, together with $<10 \mathrm{~nm}$ closeness between the donor and acceptor molecules [51]. In this regard, PFBD and PFBT (Fig. 1A) were selected as the potential FRET donors, as their emission spectra overlapped commendably with the PFDBD10 absorption spectrum (Fig. 2A). The donor-acceptor pairs were concurrently encapsulated into lipid-PEG nanoparticles, affording PFBD/PFDBD10-SPNs and PFBT/PFDBD10-SPNs, respectively. The space constraint within the nanoparticle made the donor and acceptor SPs in close distance, allowing for the occurrence of effective FRET.

In addition, the formation and self-assembly process of the SPNs were monitored by dynamic light scattering (DLS). Taking PFBD/ PFDBD10-SPNs for example, PFBD, PFDBD10, as well as lipid-PEG were dissolved in $1 \mathrm{~mL}$ of THF. DLS measurement indicated that such THF solution had a count rate of $47.0 \mathrm{kcps}$. Subsequently, $1 \mathrm{~mL}$ of the THF solution was dropped into $9 \mathrm{~mL}$ of water under sonication using a microtip probe sonicator. After further sonication of the mixture for another $60 \mathrm{~s}$ followed by evaporation of THF, the resultant aqueous suspension was also measured by DLS. Noteworthy, the count rate of the aqueous suspension significantly increased to $258.1 \mathrm{kcps}$, suggesting the formation of self-assembled micelles.

\subsection{Highly amplified NIR fluorescence within SPNs}

To modulate the enhancement of NIR fluorescence, PFBD/ PFDBD10-SPNs and PFBT/PFDBD10-SPNs with different molar ratios between the repeat units (RU) of donor and acceptor ([donor $\mathrm{RU}] /[$ acceptor RU]) that ranged from $0: 1$ to $2.4: 1$ (with fixed PFDBD10 loading content) were fabricated. DLS data indicated that PFBD/PFDBD10-SPNs and PFBT/PFDBD10-SPNs had similar particle size at each [donor RU]/[acceptor RU], which increased from $\sim 63$ to $\sim 90 \mathrm{~nm}$ with [donor $\mathrm{RU}] /[$ acceptor RU] varied from 0:1 to 2.4:1 (Supplementary Tables S1 and S2). Transmission electron microscopy (TEM) result suggested that the obtained SPNs had spherical shape (Fig. 2B). As shown in Fig. 2C and D, dramatic FRET took place within both PFBD/PFDBD10-SPNs and PFBT/PFDBD10-SPNs, as evidenced by significant reduction of donor SP fluorescence and simultaneous enhancement of NIR emission of PFDBD10. The FRET efficiencies of PFBD/PFDBD10-SPNs and PFBT/PFDBD10-SPNs ([donor $\mathrm{RU}] /[$ acceptor $\mathrm{RU}]=2.4: 1$ ) were both $>95 \%$ through quantifying the quenching of donor emission, revealing the efficient FRET. The amplification of PFDBD10 emission was determined by comparing the fluorescence of SPNs containing donor-acceptor pair with that of PFDBD10-SPNs, upon excitation at absorption maximum of donors, that was, $475 \mathrm{~nm}$ for PFBD and $460 \mathrm{~nm}$ for PFBT. Noteworthy, as summarized in Supplementary Tables S1 and S2, the NIR emission of PFDBD10 was able to be amplified for 53.7 and 35.9 folds within the PFBD/PFDBD10-SPNs and PFBT/PFDBD10SPNs, respectively, at [donor RU]/[acceptor RU] $=2.4: 1$.

The fluorescence life-times of donor SP within SPNs containing donor-acceptor pair (2.4:1) and only donor (2.4:0) were also investigated. The results displayed in Fig. $2 \mathrm{E}$ and $\mathrm{F}$ as well as Supplementary Tables S3 and S4 indicated that the life-time of the excited state of donor within donor-acceptor pair co-loaded SPNs was significantly decreased as compared to that of donor within SPNs containing only donor SP, which confirmed the occurrence of FRET in both PFBD/PFDBD10-SPNs and PFBT/PFDBD10-SPNs.
A

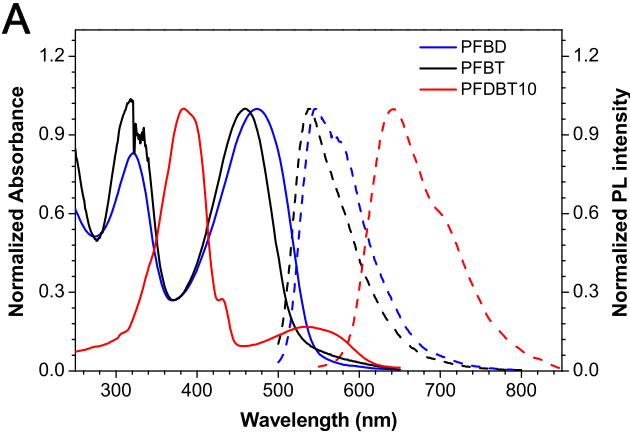

B

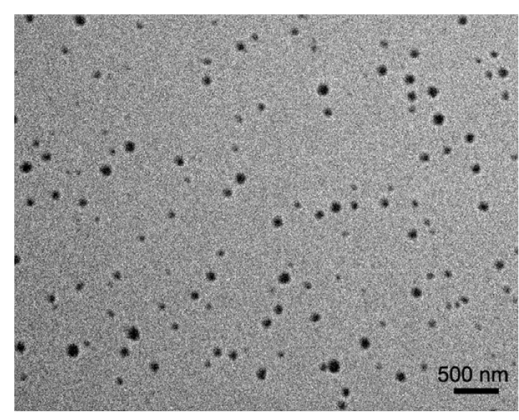

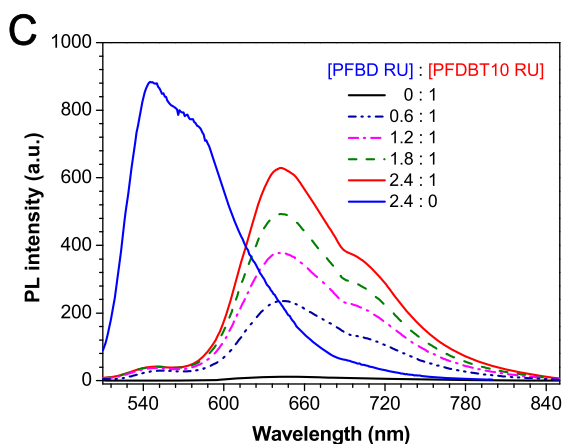

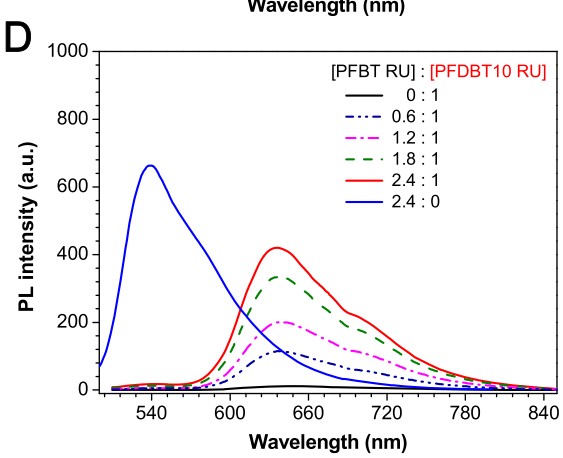

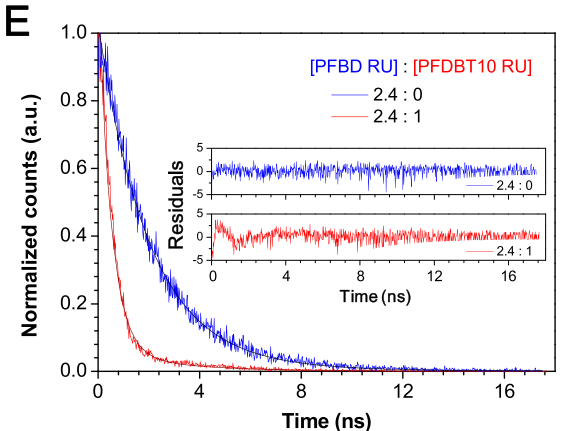

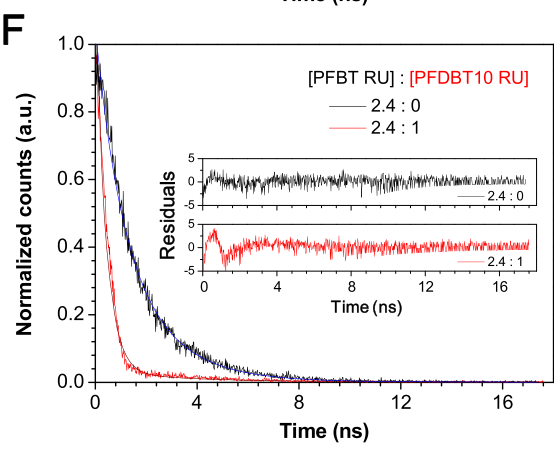

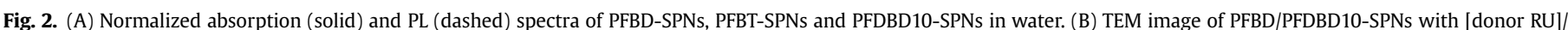

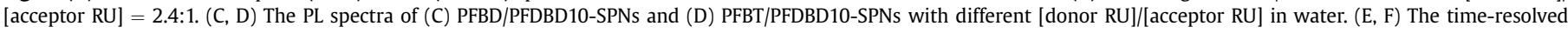
fluorescence decay of donor within SPNs containing donor-acceptor pair (2.4:1) and only donor (2.4:0). Donor: PFBD for (E) and PFBT for (F). 
Thanks to the intra-particle energy transfer, the PFBD/PFDBD10SPNs and PFBT/PFDBD10-SPNs with [donor RU]/[acceptor $\mathrm{RU}]=2.4: 1$ exhibited the PL QY of 0.47 and 0.32 in water, respectively, using 4-(dicyanomethylene)-2-methyl-6-(p-dimethylaminostyryl)-4H-pyran in methanol $(0.43)$ as the standard. To the best of our knowledge, the QY of 0.47 for PFBD/PFDBD10-SPNs could be referred to as ultrahigh compared with the existing NIR fluorescent SPNs and inorganic QDs.

It was obvious that PFBD was a better FRET donor than PFBT to fabricate FRET-SPNs, as PFBD could provide larger molar absorptivity at $488 \mathrm{~nm}$ (equipped laser excitation of the confocal microscope; Supplementary Fig. S2), greater amplification of acceptor emission, and higher PL QY of FRET-SPNs in water. Therefore, PFBD/ PFDBD10-SPNs with [donor RU]/[acceptor RU] $=2.4: 1$ were employed for the next EC tracking experiments. To improve the uptake by ECs, PFBD/PFDBD10-SPNs were further functionalized with an EC proteoglycan-binding peptide (PBP) via the coupling reaction between the maleimide group of SPN and the thiol group of PBP, yielding PFBD/PFDBD10-PBP-SPNs (Fig. 1B). The zeta potential investigation revealed that PFBD/PFDBD10-SPNs had a negative zeta potential of $-26.9 \mathrm{mV}$, which changed to a positive one $(21.8 \mathrm{mV})$ after reaction with PBPs. As PBP contained a number of positively charged amino acids, this result indicated the successful surface functionalization of PFBD/PFDBD10-SPNs. It was estimated that $\sim 6000$ EC PBPs were conjugated on each SPN on average. We demonstrated that the EC uptake of SPNs was significantly improved by the modification with PBPs (Supplementary Fig. S3), by virtue of the tight binding of PBPs to proteoglycans on the surface of ECs [52].

\subsection{In vitro EC tracking and vasculogenic potential}

The cytotoxicity study of PFBD/PFDBD10-PBP-SPNs against ECs was carried out. As depicted in Supplementary Fig. S4, the EC viabilities after incubation with $5 \mathrm{nM}$ ( 5 folds higher than that used for in vitro and in vivo EC tracking studies) of PFBD/PFDBD10-PBP-SPNs were all above $95 \%$ throughout 10-day tested duration, suggesting that PFBD/PFDBD10-PBP-SPNs were almost non-toxic against ECs and suitable for EC tracking application. Next, the application of PFBD/PFDBD10-PBP-SPNs in in vitro EC tracking was studied. A commercial QD-based cell tracker, Qtracker ${ }^{\circledR} 655$, was used as a reference. After simple incubation with PFBD/PFDBD10-PBP-SPNs $(1 \mathrm{nM})$ at $37^{\circ} \mathrm{C}$ for $4 \mathrm{~h}$, the ECs were subcultured for 1,5 , and 10 days, respectively. At each time point, the fluorescence of PFBD/ PFDBD10-PBP-SPN-labeled ECs was monitored by confocal laser scanning microscopy (CLSM) and flow cytometry. As displayed in Fig. 3A and Supplementary Fig. S5A, the fluorescent SPNs were mainly distributed in the cytoplasm around the nucleus of ECs. Although the number and fluorescence intensity of PFBD/PFDBD10PBP-SPNs within the ECs were decreased over time, significantly bright fluorescence signals could still be observed in most of the cells even post 10-day subculture. The flow cytometry result revealed that the EC labeling rates of PFBD/PFDBD10-PBP-SPNs were $97.4 \%, 91.8 \%$, and $70.5 \%$ upon subculture for 1,5 , and 10 days, respectively (Fig. 3B). As a control, Qtracker ${ }^{\mathbb{B}} 655$ was also used to label ECs with the same concentration and experimental procedures as that for PFBD/PFDBD10-PBP-SPNs. Noteworthy, the fluorescence intensity from Qtracker ${ }^{\circledR}$ 655-labeled ECs was obviously lower than that from PFBD/PFDBD10-PBP-SPN-labeled cells at each time point, indicated by both CLSM and flow cytometry data (Fig. 3A and B and Supplementary Fig. S5). Quantitatively, on day 1, the mean fluorescence intensity from PFBD/PFDBD10-PBP-SPNlabeled ECs was $~ 12.0$-fold higher than that from Qtracker ${ }^{\circledR} 655$ labeled ones, analyzed by flow cytometry. Additionally, the EC labeling rates for Qtracker $^{\circledR} 655$ were $84.5 \%, 62.1 \%$, and $14.8 \%$ after 1 ,
5 , and 10 days subculture, respectively. The value was also lower than that for PFBD/PFDBD10-PBP-SPNs at each time point of subculture. These results verified that PFBD/PFDBD10-PBP-SPNs gave better performance in in vitro EC tracing compared with Qtracker ${ }^{\circledR}$ 655 , which was known as one of the most effective long-term fluorescent cell trackers [53].

It was known that ECs possessed angiogenic potential, that was, could form capillary-like tubes in vitro [54]. To investigate whether PFBD/PFDBD10-PBP-SPNs labeling affects such function of ECs, tube formation assay was carried out. As shown in Fig. 4A and B, PFBD/ PFDBD10-PBP-SPN-labeled ECs with bright NIR fluorescence were able to assemble into capillary-like tube structures. This agreed well with what untreated ECs formed in terms of size, pattern and density of tubes (Fig. 4C). Quantitative analysis suggested that there was no significant difference in the number of tubes at $24 \mathrm{~h}$ between the groups of ECs with and without labeling of PFBD/ PFDBD10-PBP-SPNs (Fig. 4D). The result suggested that the SPN labeling had negligible interference on the vasculogenic potential of ECs.

\subsection{In vivo EC tracking post systemic infusion}

Prior to in vivo EC tracking investigation, the in vivo biodistribution, metabolization and toxicity of PFBD/PFDBD10-PBPSPNs were conducted. After intravenous administration of PFBD/ PFDBD10-PBP-SPNs (50 nM, $0.1 \mathrm{~mL}$ ), the healthy mice were sacrificed at designated time intervals, followed by excision and ex vivo fluorescence imaging of various tissues including liver, spleen, kidney, heart, lung, intestine, and stomach. It was found that the fluorescent signal was mainly distributed in liver organ after intravenous injection for 1 day, indicating that most of the PFBD/ PFDBD10-PBP-SPNs were accumulated in the liver. The fluorescent signal from PFBD/PFDBD10-PBP-SPNs decreased as the time elapsed, which was almost vanished on day 7 post-injection (Supplementary Fig. S6A). Furthermore, obvious PFBD/PFDBD10PBP-SPN fluorescence was observed in the collected feces within the initial 3 days, implying that PFBD/PFDBD10-PBP-SPNs were able to be easily excreted from the body by biliary pathway from liver, bile duct to feces (Supplementary Fig. S6B). Subsequently, the potential in vivo toxicity of PFBD/PFDBD10-PBP-SPNs was investigated. After intravenous injection of PFBD/PFDBD10-PBP-SPNs ( $50 \mathrm{nM}, 0.1 \mathrm{~mL}$ ) into healthy mice for 7 days, the blood chemistry tests regarding to total bilirubin, alkaline phosphatase, $\gamma$-globulin transferase, aspartic acid transaminase, albumin, and alanine transferase were studied and compared with those from untreated healthy mice. As displayed in Supplementary Fig. S7A, there was no difference in any of the parameters between PFBD/PFDBD10-PBPSPN-treated and untreated groups, revealing that PFBD/PFDBD10PBP-SPNs had negligible harmness to liver function in spite of their significant accumulation, which might be benefitted from the relatively rapid clearance of the nanoprobes from the mouse body. Finally, the histological analyses of liver and kidney tissues indicated that PFBD/PFDBD10-PBP-SPNs did not cause pathological lesions to the organs (Supplementary Fig. S7B). The results reasonably demonstrated that PFBD/PFDBD10-PBP-SPNs possessed low in vivo toxicity.

Next, a CLI-bearing mouse model was established to study the feasibility of PFBD/PFDBD10-PBP-SPNs in tracking ECs in vivo and revealing the contribution of ECs to CLI treatment. In this experiment, systemic infusion including intravenous (i.v.) and intraarterial (i.a.) injections of ECs was adopted, as i.v. and i.a. administrations were the most common approaches used in the clinical cell therapy [46]. After labeling with PFBD/PFDBD10-PBP-SPNs, ECs $\left(1 \times 10^{6}\right)$ were injected into CLI-bearing mice via the tail vein or femoral artery. At designated time intervals, the mice were imaged 


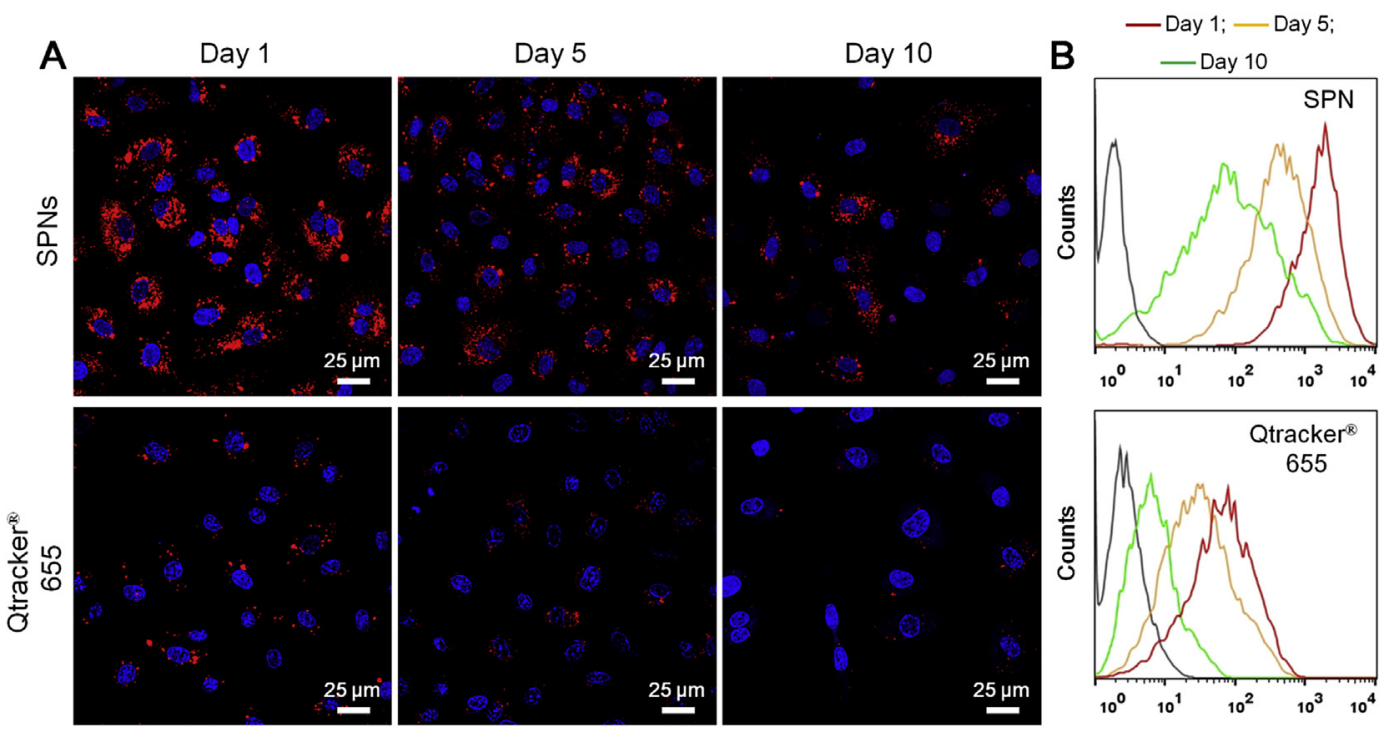

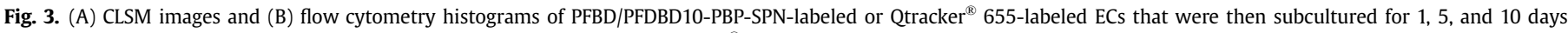

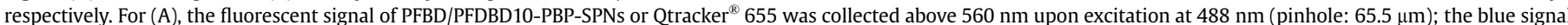

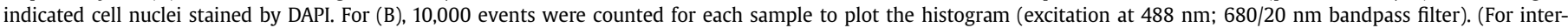
pretation of the references to colour in this figure legend, the reader is referred to the web version of this article.)
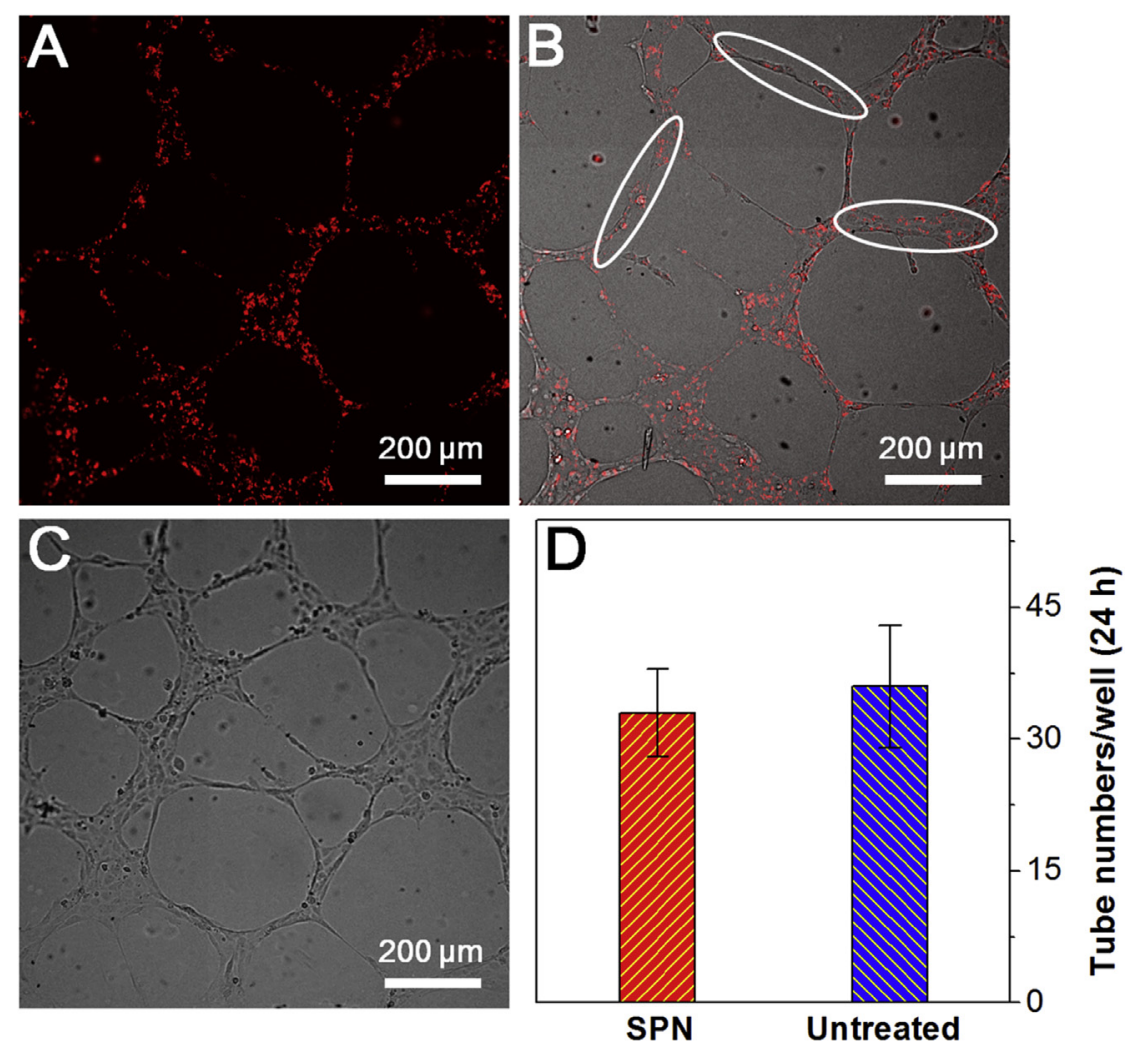

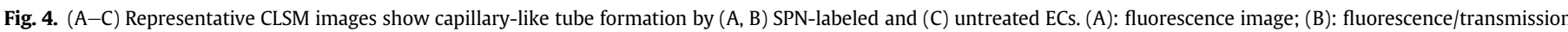

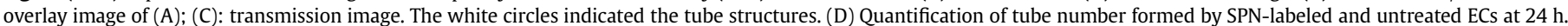

with a Maestro in vivo fluorescence imaging system. As shown in Fig. 5A, negligible fluorescence signal from PFBD/PFDBD10-PBPSPN-labeled ECs was detected in the ischemic limb (left hind limb) on day 1 post i.v. injection, which however progressively elevated and reached a maximum on day 7 . This result revealed that with the help of SPN labeling, it was visible to monitor the continued capacity of ECs to home and engraft to the ischemic limb. Although the signal was reduced over time after 7 days, obvious fluorescence could still be observed on day 21 post-injection, indicating the long-term EC tracking capacity of PFBD/PFDBD10-PBP-SPNs. In comparison, there was few fluorescence signal in the normal leg (right hind limb) throughout 21-day monitoring period (Fig. 5A), 

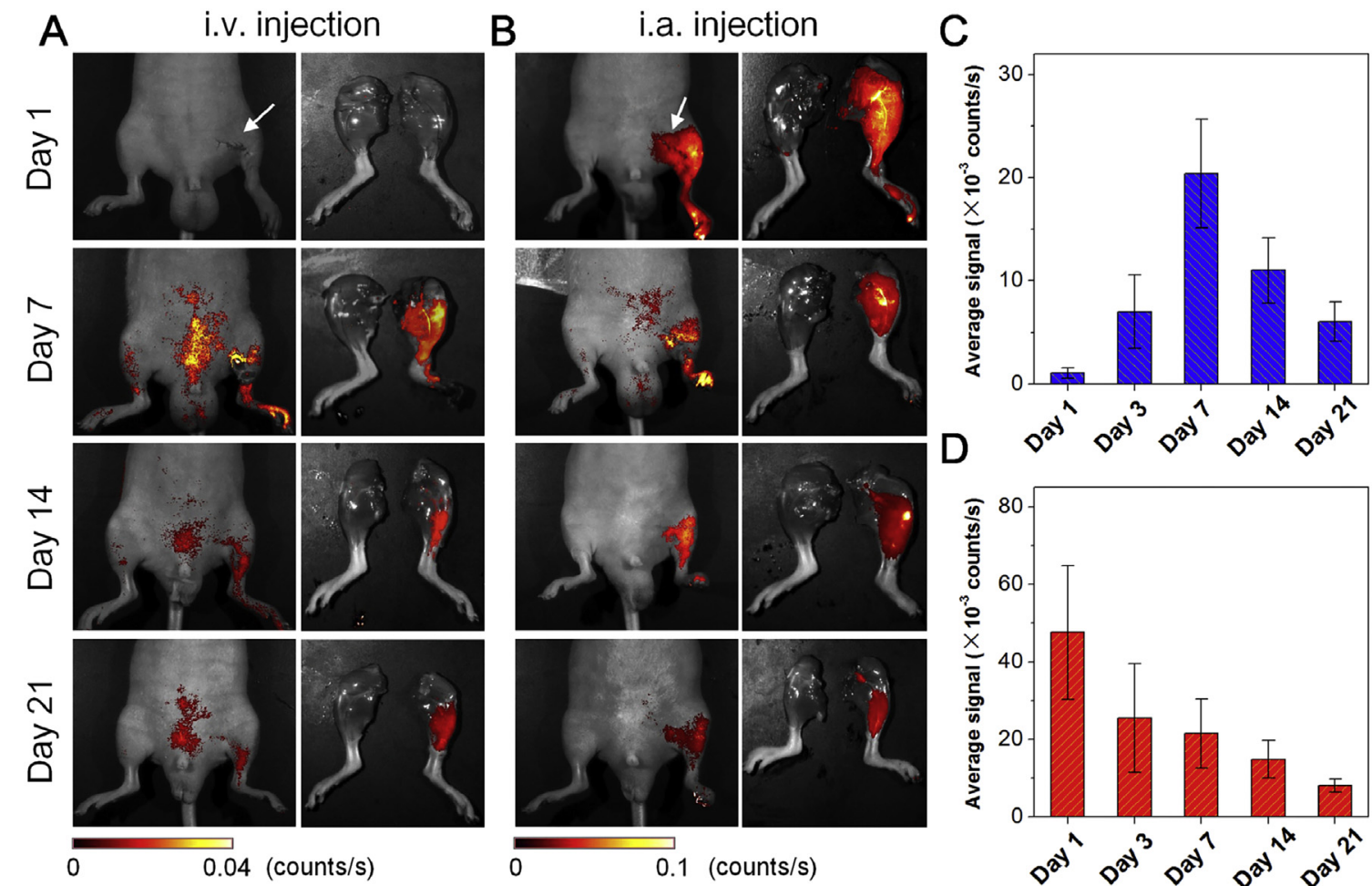

0.1 (counts/s)
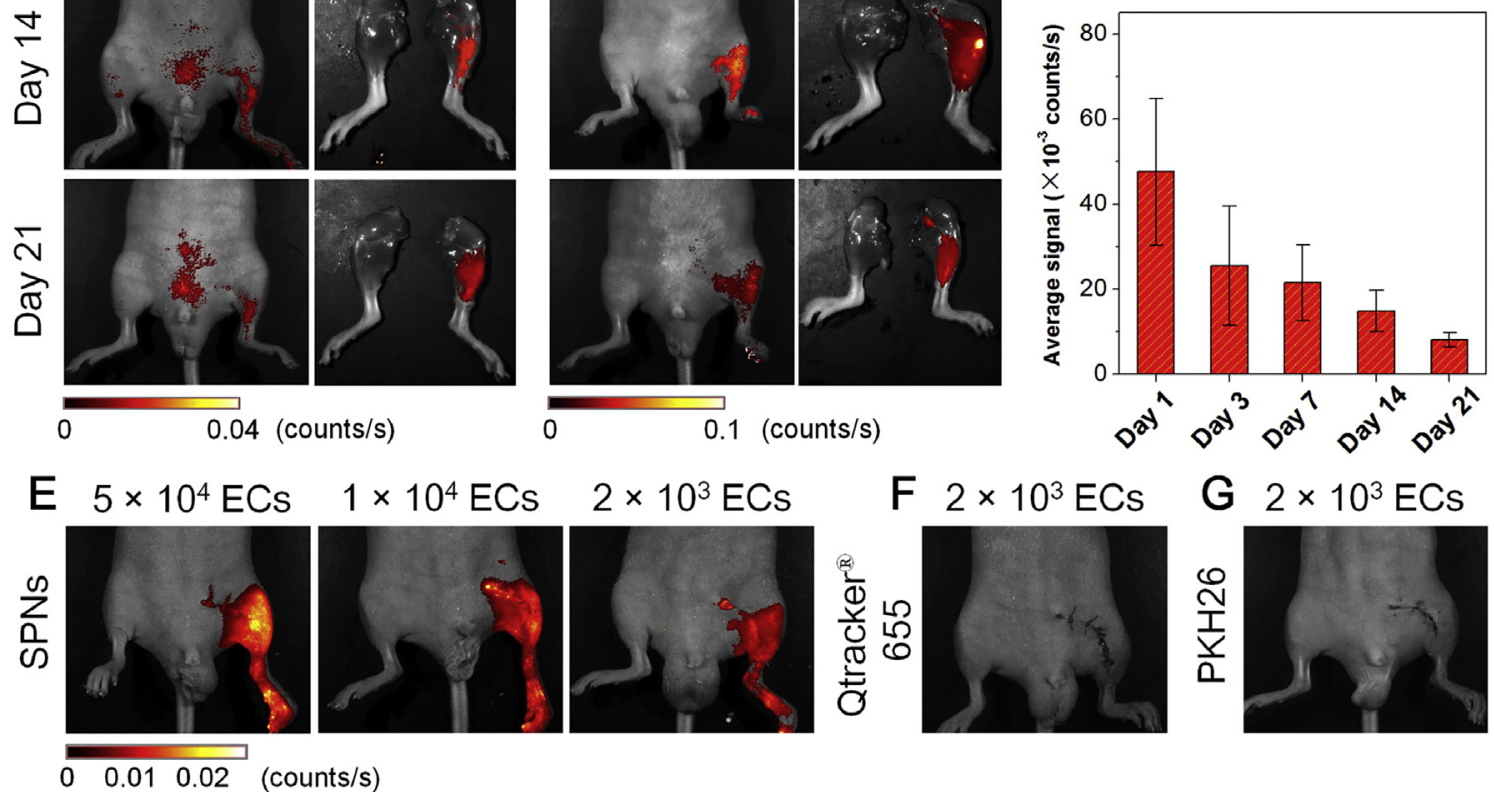

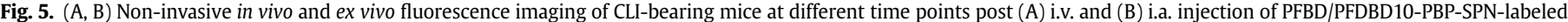

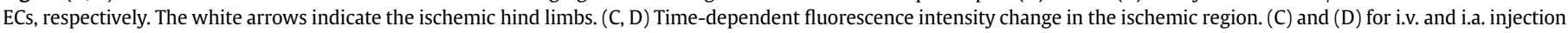

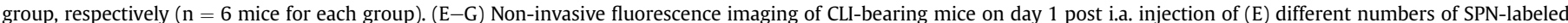

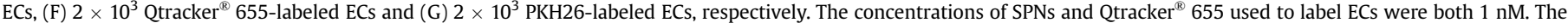
recommended working concentration of $2 \mu \mathrm{M}$ was adopted for PKH26 to label ECs.

verifying the good selectivity of i.v. injected ECs toward ischemic limb. The biodistribution and metabolization of i.v. injected ECs in the major normal tissues were also examined by taking advantage of SPN labeling (Supplementary Fig. S8). On day 1, ECs were actively trapped in lung rather than other tissues because of the pulmonary first-pass effect [55]. The fluorescence intensity in lung was significantly decreased as the time elapses, and only a few fluorescence signals could be detected in lung post 7 days. Additionally, besides ischemic limb, relatively strong fluorescence signal from SPN-labeled ECs was observed as well in the mouse intestine on day 7 after i.v. injection, which was then decreased over time. As a control, the CLI-bearing mice i.v. injected with saline were also imaged under the same experiment conditions as those for Fig. 5A. It was noted that there was no fluorescent signal observed in the ischemic limb and any of normal organs within 7-day tested duration (Supplementary Fig. S9), revealing that the fluorescent signals in Fig. 5A and Supplementary Fig. S8 indeed came from the SPNs.

On the other hand, when changing the injection method to i.a., rather intense fluorescence signal could be seen in the ischemic limb on day 1 , which gradually decreased thereafter (Fig. 5B). This result indicated that SPN-labeled ECs through i.a. injection could engraft to the ischemic region more rapidly as compared to those via i.v. injection. The quantitative analysis displayed in Fig. 5 C and D not only indicated the alterations of fluorescence signal within 21day study duration for each injection method, but also revealed that much more ECs could be enriched in the ischemic leg by i.a. injection when compared the maximal fluorescence intensity in the ischemic leg between i.a. (day 1) and i.v. (day 7) injections. Moreover, the biodistribution data in Supplementary Fig. S10 suggested that on day 1 post i.a. injection, SPN-labeled ECs did not accumulate in the lung tissue, but engrafted to intestine instead. After 7 days, the fluorescence signal from SPN-labeled ECs in the intestine was almost completely eliminated, leaving the exclusive signal in the ischemic limb.

\subsection{Sensitivity and accuracy of SPNs in tracking ECs in vivo}

To investigate the sensitivity of PFBD/PFDBD10-PBP-SPNs in reporting the fate of ECs in vivo, CLI-bearing mice were i.a. injected with different numbers of SPN-labeled ECs, respectively, followed by non-invasive imaging on day 1 post-injection. As depicted in Fig. 5E, after i.a. injection of $5 \times 10^{4}, 1 \times 10^{4}$, and $2 \times 10^{3}$ SPNlabeled ECs into mice, respectively, outstanding fluorescent signal 
could be observed in the ischemic limb. However, there was only weak fluorescence detected in the ischemic region when $1 \times 10^{3}$ SPN-labeled ECs were i.a. injected. Hence, PFBD/PFDBD10-PBPSPNs showed the limitation of tracking $2 \times 10^{3}$ i.a. injected ECs, although only a portion of $2 \times 10^{3}$ ECs could reach the target. As controls, two popularly used commercial fluorescent cell trackers, PKH26 and Qtracker ${ }^{\circledR} 655$, were used to label ECs, respectively, under the same experimental condition as that for PFBD/PFDBD10PBP-SPNs. In sharp comparison, no signal was detected in the ischemic limb after i.a. injection of $2 \times 10^{3}$ PKH26-labeled or Qtracker ${ }^{\circledR}$ 655-labeled ECs into CLI-bearing mice for 1 day (Fig. $5 \mathrm{~F}$ and $G$ ). To the best of our knowledge, for non-invasive in vivo fluorescence imaging, $2 \times 10^{3}$ represented the smallest value of systemically administered cells that could be visualized by fluorescent cell trackers. These data demonstrated the ultrahigh sensitivity of PFBD/PFDBD10-PBP-SPN in tracking ECs in vivo, which highlighted the importance of our probe design.

Furthermore, we evaluated whether PFBD/PFDBD10-PBP-SPNs could precisely track the injected ECs in vivo over a long period of time. To this end, the mice received i.a. injection of SPN-labeled ECs $\left(1 \times 10^{6}\right)$ were sacrificed on day 21 and the ischemic limbs were excised and sliced. The slices were subsequently immunostained with human nuclei antibody and 4',6-diamidino-2-phenylindole (DAPI), respectively. As the ECs used in this work originated from human umbilical vein, the nuclei of the injected ECs could be selectively visualized by human nuclei antibody [56]. DAPI was utilized to stain all the cell nuclei in the slices including the injected ECs and other mouse cells. As shown in Fig. 6A and B, the nuclei of injected human ECs (green color) were clearly seen in the slices of mouse ischemic limbs on day 21. Dramatically, almost all the fluorescence signals (red color) from PFBD/PFDBD10-PBP-SPNs were located around the green nuclei of injected ECs. Quantitatively, 92\% of injected ECs remained labeled with SPNs on day 21, which were based on 12 images from various areas of ischemic limbs $(n=4)$. Moreover, macrophages in the ischemic limb slices from mice after i.a. injection of SPN-labeled ECs for 21 days were stained against CD68 with green color. As depicted in Fig. 6C, nearly no SPNs were co-localized with macrophages, suggesting that there was negligible SPNs engulfment by resident macrophages [11]. These results together verified that our SPNs could truly report the fate of injected ECs in vivo for at least 21 days.

\subsection{Understanding regenerative contribution of ECS via SPNs}

Laser Doppler imaging was conducted to assess the treatment efficacy of SPN-labeled ECs on CLI disease, which could clearly visualize the patency status of blood flow in normal and ischemic limbs (Supplementary Fig. S11). Most of the mice received saline treatment lost the left foot or limb due to the severe CLI. Nevertheless, on day 21 after i.v. and i.a. injection of SPN-labeled ECs, respectively, important vascularization in the left hind limbs of mice was observed (Fig. 7A), suggesting that systemic infusion of SPN-labeled ECs significantly promoted tissue repair. It was also found that the perfusion ratio and percentage of limb salvage for i.a. injection group were higher than that for i.v. injection cohort (Fig. 7B and C), which should be owing to the larger number of ECs enriched in the ischemic limb by i.a. administration.

To understand the dedication of ECs in CLI therapy, the blood vessels in the ischemic limbs of mice in i.a. and i.v. injection groups were immunostained against CD31 on day 21. Significantly high density of CD31-positive blood vessels was observed in the slices of ischemic limbs for two ECs treatment groups. More importantly, the CLSM images at different vertical depths (Fig. 8A and Supplementary Figs. S12 and 3D sectional image (Fig. 8B) displayed that i.a. or i.v. injected SPN-labeled ECs (red color) were located exactly in the blood vessels (green color), revealing that ECs took part in neovascularisation via forming vascular structures. Therefore, by virtue of SPN labeling, it was reasonable to say that participating in angiogenesis in vivo was an important contribution of ECs to CLI therapy.

\section{Conclusions}

In summary, we employed FRET strategy to develop and optimize a highly NIR emissive SPN for long-term tracking of systemically administered ECs in CLI-bearing mice. Through comparison of two different donor SPs, it was found that PFBD gave better performance as the FRET donor due to higher absorptivity at $488 \mathrm{~nm}$, larger enhancement of acceptor emission, and higher QY of SPNs in water (as high as 0.47). In vivo studies verified that PFBD/PFDBD10PBP-SPNs could serve as a unique nanoprobe to provide precise information on the in vivo behaviors of systemically injected ECs in CLI treatment for at least 21 days. Moreover, PFBD/PFDBD10-PBPSPNs were capable of effectively tracking as few as $2 \times 10^{3}$ ECs upon i.a. injection into CLI-bearing mice, showing much higher sensitivity in ECs tracking in vivo compared with commercial Qtracker ${ }^{\circledR}$ 655 and PKH26. By virtue of SPN labeling, it was also found that ECs contributed to CLI treatment by participating in angiogenesis. Our PFBD/PFDBD10-PBP-SPNs thus met nearly all the necessary requirements of an advanced fluorescent cell tracker, including ultrahigh NIR fluorescence, superb biological and photophysical stabilities, negligible interference on cell behavior and function,
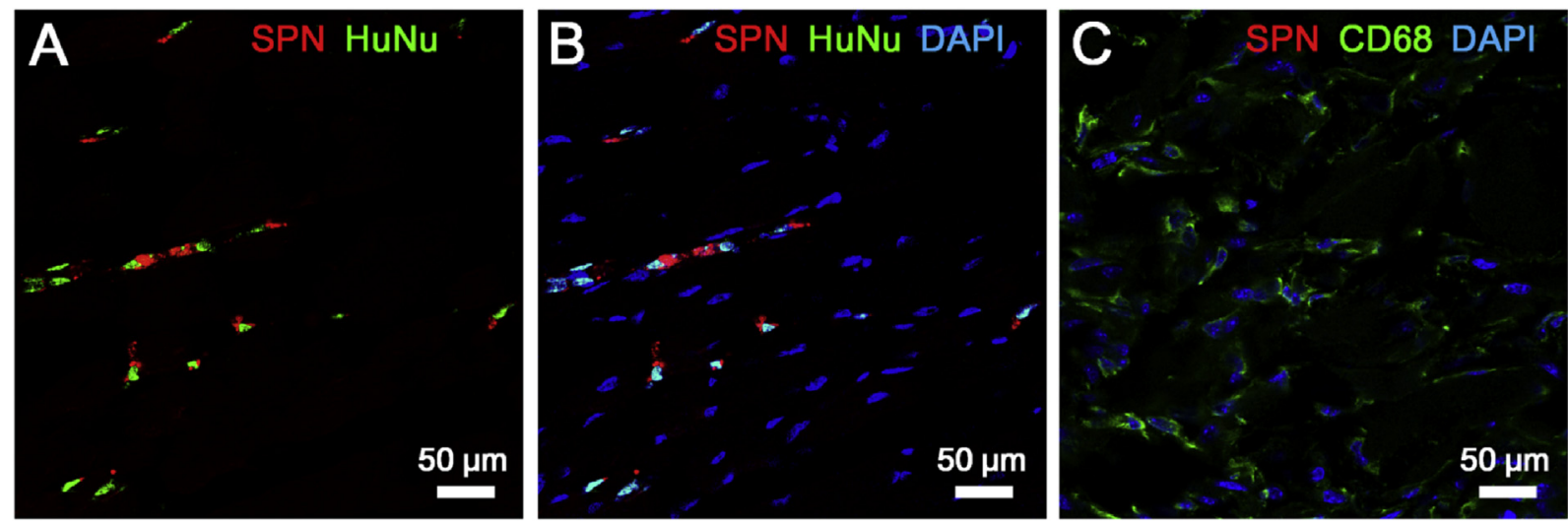

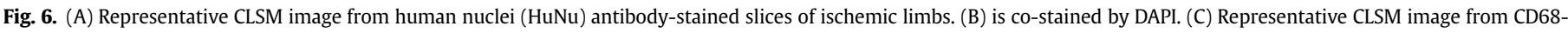
staining slices of ischemic limbs. For $(A-C)$, the mice were sacrificed on day 21 post i.a. injection of SPN-labeled ECs. 

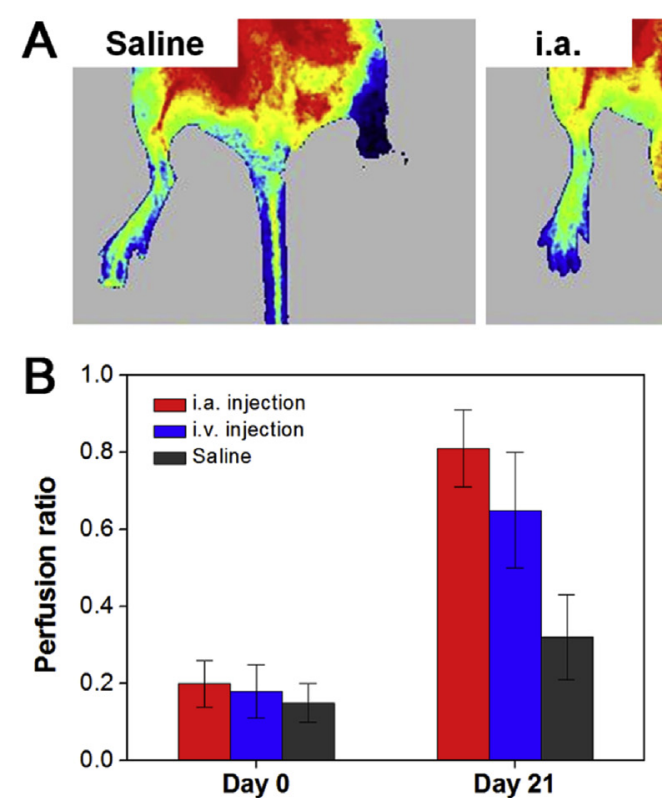

i.a.

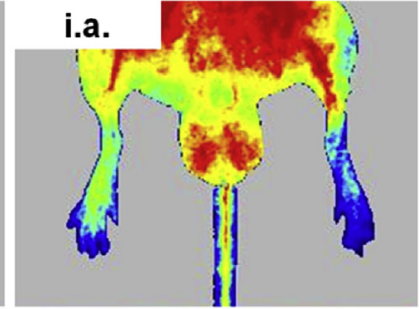

C

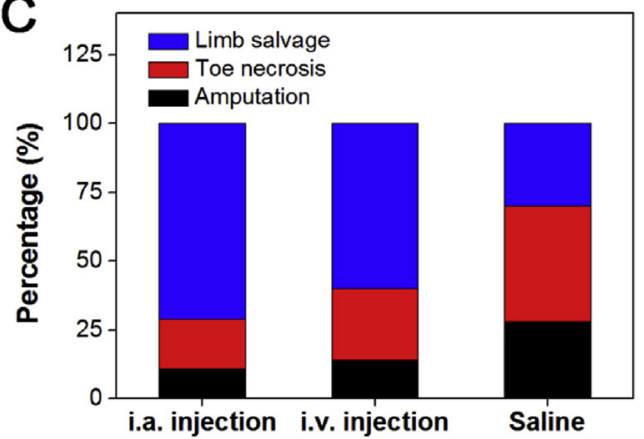

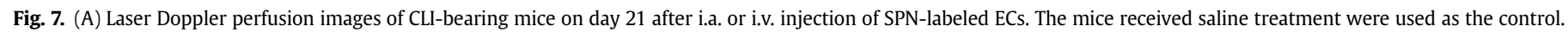
(B) Quantification of perfusion recovery based on Laser Doppler imaging. (C) Determination of limb/foot status of CLI-bearing mice in various treatment groups on day 21.

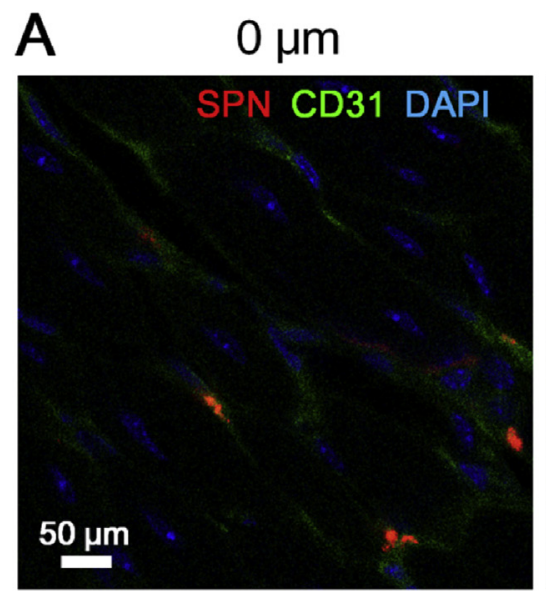

$4.5 \mu \mathrm{m}$
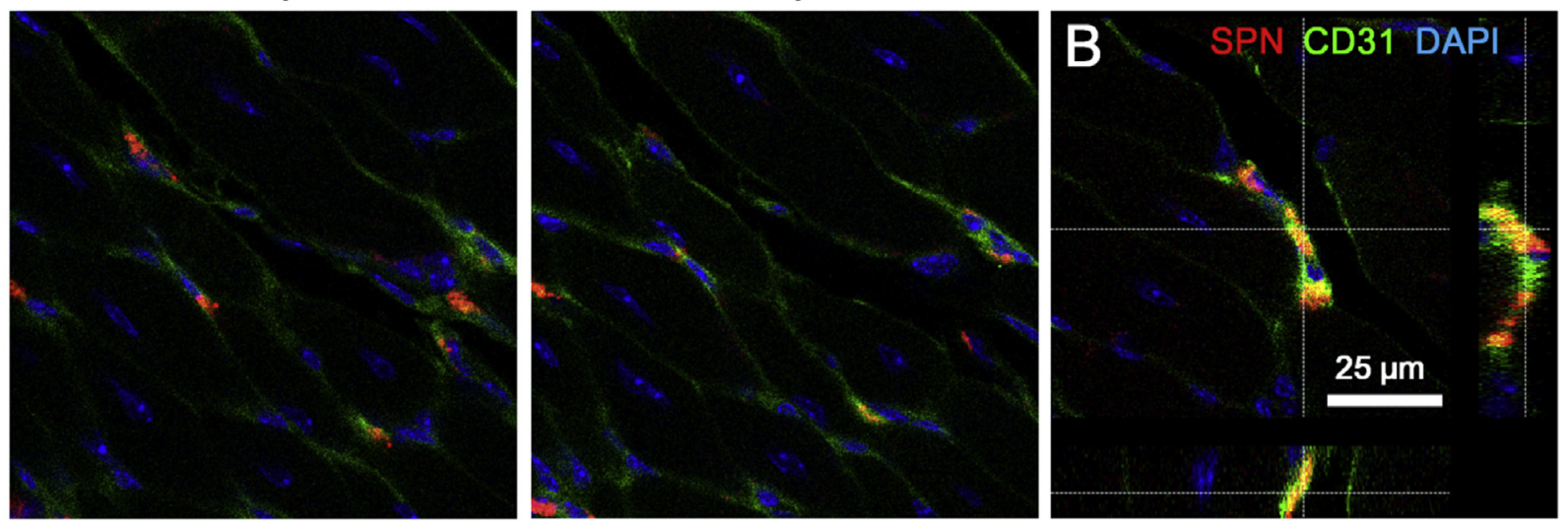

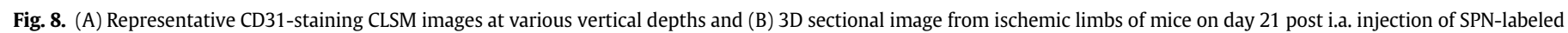
ECs. 
excellent retention within cells, as well as precise long-term cell tracking ability. This work will inspire new insights and materials in the field of cell tracking.

\section{Conflict of interest}

Authors declare no conflict of interest.

\section{Acknowledgments}

This work was supported by the NSFC (81220108015, 51622305, 31571011, 81670624, and 21402219), the PCSIRT (IRT13023), the National Basic Research Program of China (2015CB856503), the 111 project (B08011) and the Science \& Technology Project of Tianjin of China (No. 15JCYBJC29800).

\section{Appendix A. Supplementary data}

Supplementary data related to this article can be found at http:// dx.doi.org/10.1016/j.biomaterials.2017.07.038.

\section{References}

[1] F. Arslan, D.P. de Kleijn, G. Pasterkamp, Innate immune signaling in cardiac ischemia, Nat. Rev. Cardiol. 8 (2011) 292-300.

[2] P.J. Chen, Y.D. Kang, C.H. Lin, S.Y. Chen, C.H. Hsieh, Y.Y. Chen, et al., Multitheragnostic multi-GNRs crystal-seeded magnetic nanoseaurchin for enhanced in vivo mesenchymal-stem-cell homing, multimodal imaging, and stroke therapy, Adv. Mater. 27 (2015) 6488-6495.

[3] R.M. Bober, E. Jahangir, What is ischemia and how should this be defined based on modern imaging, Prog. Cardiovasc. Dis. 57 (2015) 537-554.

[4] S. Yamazoe, M. Naya, M. Shiota, T. Morikawa, A. Kubo, T. Tani, et al., Large-area surface-enhanced Raman spectroscopy imaging of brain ischemia by gold nanoparticles grown on random nanoarrays of transparent boehmite, ACS Nano 8 (2014) 5622-5632.

[5] M. Teraa, M.S. Conte, F.L. Moll, M.C. Verhaar, Critical limb ischemia: current trends and future directions, J. Am. Heart Assoc. 5 (2016) e002938.

[6] C. Tu, S. Das, A.B. Baker, J. Zoldan, L.J. Suggs, Nanoscale strategies: treatment for peripheral vascular disease and critical limb ischemia, ACS Nano 9 (2015) 3436-3452.

[7] L. Deveza, J. Choi, J. Lee, N. Huang, J. Cooke, F. Yang, Polymer-DNA nanoparticle-induced CXCR4 overexpression improves stem cell engraftment and tissue regeneration in a mouse hindlimb ischemia model, Theranostics 6 (2016) 1176-1189.

[8] H.N. Yang, J.S. Park, D.G. Woo, S.Y. Jeon, K.H. Park, Transfection of VEGF(165) genes into endothelial progenitor cells and in vivo imaging using quantum dots in an ischemia hind limb model, Biomaterials 33 (2012) 8670-8684.

[9] N.F. Huang, H. Niiyama, C. Peter, A. De, Y. Natkunam, F. Fleissner, et al., Embryonic stem cell-derived endothelial cells engraft into the ischemic hindlimb and restore perfusion, Arterioscler. Thromb. Vasc. Biol. 30 (2010) 984-991.

[10] W.W.W. Hsiao, Y.Y. Hui, P.C. Tsai, H.C. Chang, Fluorescent nanodiamond: a versatile tool for long-term cell tracking, super-resolution imaging, and nanoscale temperature sensing, Acc. Chem. Res. 49 (2016) 400-407.

[11] T.J. Wu, Y.K. Tzeng, W.W. Chang, C.A. Cheng, Y. Kuo, C.H. Chien, et al., Tracking the engraftment and regenerative capabilities of transplanted lung stem cells using fluorescent nanodiamonds, Nat. Nanotechnol. 8 (2013) 682-689.

[12] B. Dong, X. Song, X. Kong, C. Wang, Y. Tang, Y. Liu, et al., Simultaneous nearinfrared and two-photon in vivo imaging of $\mathrm{H}_{2} \mathrm{O}_{2}$ using a ratiometric fluorescent probe based on the unique oxidative rearrangement of oxonium, Adv. Mater. 28 (2016) 8755-8759.

[13] J.V. Frangioni, In vivo near-infrared fluorescence imaging, Curr. Opin. Chem. Biol. 7 (2003) 626-634.

[14] R. Weissleder, A clearer vision for in vivo imaging, Nat. Biotechnol. 19 (2001) $316-317$.

[15] F. Lv, Q. Tian, L. Liu, J. Ying, S. Wang, Recent advances in conjugated polymer materials for disease diagnosis, Small 12 (2016) 696-705.

[16] L. Feng, C. Zhu, H. Yuan, L. Liu, F. Lv, S. Wang, Conjugated polymer nanoparticles: preparation, properties, functionalization and biological applications, Chem. Soc. Rev. 42 (2013) 6620-6633.

[17] C. Wu, D.T. Chiu, Highly fluorescent semiconducting polymer dots for biology and medicine, Angew. Chem. Int. Ed. 52 (2013) 3086-3109.

[18] C. Zhu, L. Liu, Q. Yang, F. Lv, S. Wang, Water-soluble conjugated polymers for imaging, diagnosis, and therapy, Chem. Rev. 112 (2012) 4687-4735.

[19] A. Duarte, K. Pu, B. Liu, G.C. Bazan, Recent advances in conjugated polyelectrolytes for emerging optoelectronic applications, Chem. Mater. 23 (2011) $501-515$.

[20] Q. Miao, Y. Lyu, D. Ding, K. Pu, Semiconducting oligomer nanoparticles as an activatable photoacoustic probe with amplified brightness for in vivo imaging of pH, Adv. Mater. 28 (2016) 3662-3668.

21] K. Pu, A.J. Shuhendler, J.V. Jokerst, J. Mei, S.S. Gambhir, Z. Bao, et al., Semiconducting polymer nanoparticles as photoacoustic molecular imaging probes in living mice, Nat. Nanotechnol. 9 (2014) 233-239.

[22] G. Hong, Y. Zou, A.L. Antaris, S. Diao, D. Wu, K. Cheng, et al., Ultrafast fluorescence imaging in vivo with conjugated polymer fluorophores in the second near-infrared window, Nat. Commun. 5 (2014) 4206.

[23] H. Shi, X. Ma, Q. Zhao, B. Liu, Q. Qu, Z. An, et al., Ultrasmall phosphorescent polymer dots for ratiometric oxygen sensing and photodynamic cancer therapy, Adv. Funct. Mater. 24 (2014) 4823-4830.

[24] C. Wu, S.J. Hansen, Q. Hou, J. Yu, M. Zeigler, Y. Jin, et al., Design of highly emissive polymer dot bioconjugates for in vivo tumor targeting, Angew. Chem. Int. Ed. 50 (2011) 3430-3434.

[25] P. Howes, M. Green, J. Levitt, K. Suhling, M. Hughes, Phospholipid encapsulated semiconducting polymer nanoparticles: their use in cell imaging and protein attachment, J. Am. Chem. Soc. 132 (2010) 3989-3996.

[26] Q. Zhao, X. Zhou, T. Cao, K.Y. Zhang, L. Yang, S. Liu, et al., Fluorescent/phosphorescent dual-emissive conjugated polymer dots for hypoxia bioimaging, Chem. Sci. 6 (2015) 1825-1831.

[27] J. Pecher, S. Mecking, Nanoparticles of conjugated polymers, Chem. Rev. 110 (2010) 6260-6279.

[28] A.J. Shuhendler, K. Pu, L.N. Cui, J.P. Uetrecht, J. Rao, Real-time imaging of oxidative and nitrosative stress in the liver of live animals for drug-toxicity testing, Nat. Biotechnol. 32 (2014) 373-380.

[29] H.Y. Liu, P.J. Wu, S.Y. Kuo, C.P. Chen, E.H. Chang, C.Y. Wu, et al., Quinoxalinebased polymer dots with ultrabright red to near-infrared fluorescence for in vivo biological imaging, J. Am. Chem. Soc. 137 (2015) 10420-10429.

[30] P.J. Wu, S.Y. Kuo, Y.C. Huang, C.P. Chen, Y.H. Chan, Polydiacetylene-enclosed near-infrared fluorescent semiconducting polymer dots for bioimaging and sensing, Anal. Chem. 86 (2014) 4831-4839.

[31] K. Li, D. Ding, D. Huo, K. Pu, N.P.T. Ngo, Y. Hu, et al., Conjugated polymer based nanoparticles as dual-modal probes for targeted in vivo fluorescence and magnetic resonance imaging, Adv. Funct. Mater. 22 (2012) 3107-3115.

[32] K. Yang, H. Xu, L. Cheng, C. Sun, J. Wang, Z. Liu, In vitro and in vivo nearinfrared photothermal therapy of cancer using polypyrrole organic nanoparticles, Adv. Mater. 24 (2012) 5586-5592.

[33] D, Zhang M. Wu, Y.Y. Zeng, N.S, Liao, ZX. Cai, G, Liu, et al, Lipid micelles packaged with semiconducting polymer dots as simultaneous MRI/photoacoustic imaging and photodynamic/photothermal dual-modal therapeutic agents for liver cancer, J. Mater. Chem. B 4 (2016) 589-599.

[34] K. Sun, Y. Tang, O. Li, S. Yin, W. Oin, J. Yu, et al., In vivo dynamic monitoring of small molecules with implantable polymer-dot transducer, ACS Nano 10 (2016) 6769-6781.

[35] X. Zhen, C. Zhang, Q. Miao, K.L. Lim, K. Pu, Intraparticle energy level alignment of semiconducting polymer nanoparticles to amplify chemiluminescence for ultrasensitive in vivo imaging of reactive oxygen species, ACS Nano 10 (2016) 6400-6409.

[36] X. Gong, M.H. Tong, S.H. Park, M. Liu, A. Jen, A.J. Heeger, Semiconducting polymer photodetectors with electron and hole blocking layers: high detectivity in the near-infrared, Sensors 10 (2010) 6488-6496.

[37] G. Jin, D. Mao, P. Cai, R. Liu, N. Tomczak, J. Liu, et al, Conjugated polyme nanodots as ultrastable long-term trackers to understand mesenchymal stem cell therapy in skin regeneration, Adv. Funct. Mater. 25 (2015) 4263-4273.

[38] H. Yuan, J. Qi, C. Xing, H. An, R. Niu, Y. Zhan, et al., Graphene-oxide-conjugated polymer hybrid materials for calmodulin sensing by using FRET strategy, Adv. Funct. Mater. 25 (2015) 4412-4418.

[39] L. Cheng, K. Yang, Q. Chen, Z. Liu, Organic stealth nanoparticles for highly effective in vivo near-infrared photothermal therapy of cancer, ACS Nano 6 (2012) 5605-5613.

[40] H.S. Peng, D.T. Chiu, Soft fluorescent nanomaterials for biological and biomedical imaging, Chem. Soc. Rev. 44 (2015) 4699-4722.

[41] C.T. Kuo, A.M. Thompson, M.E. Gallina, F. Ye, E.S. Johnson, W. Sun, et al., Optical painting and fluorescence activated sorting of single adherent cells labelled with photoswitchable Pdots, Nat. Commun. 7 (2016) 11468.

[42] K. Li, B. Liu, Polymer-encapsulated organic nanoparticles for fluorescence and photoacoustic imaging, Chem. Soc. Rev. 43 (2014) 6570-6597.

[43] D. Ding, J. Liu, G. Feng, K. Li, Y. Hu, B. Liu, Bright far-red/near-infrared conjugated polymer nanoparticles for in vivo bioimaging, Small 9 (2013) 3093-3102.

[44] C. Wu, T. Schneider, M. Zeigler, J. Yu, P.G. Schiro, D.R. Burnham, et al., Bioconjugation of ultrabright semiconducting polymer dots for specific cellular targeting, J. Am. Chem. Soc. 132 (2010) 15410-15417.

[45] Y. Lyu, C. Xie, S.A. Chechetka, E. Miyako, K. Pu, Semiconducting polymer nanobioconjugates for targeted photothermal activation of neurons, J. Am. Chem. Soc. 138 (2016) 9049-9052.

[46] P. Lin, D. Correa, T.J. Kean, A. Awadallah, J.E. Dennis, A.I. Caplan, Serial transplantation and long-term engraftment of intra-arterially delivered clonally derived mesenchymal stem cells to injured bone marrow, Mol. Ther. 22 (2014) 160-168.

[47] P.K. Nguyen, J. Riegler, J.C. Wu, Stem cell imaging: from bench to bedside, Cell Stem Cell 14 (2014) 431-444.

[48] M.F. Kircher, S.S. Gambhir, J. Grimm, Noninvasive cell-tracking methods, Nat Rev. Clin. Oncol. 8 (2011) 677-688.

[49] C.Y. Fang, V. Vaijayanthimala, C.A. Cheng, S.H. Yeh, C.F. Chang, C.L. Li, et al., The exocytosis of fluorescent nanodiamond and its use as a long-term cell tracker 
Small 7 (2011) 3363-3370.

[50] L. Ferreira, J.M. Karp, L. Nobre, R. Langer, New opportunities: the use of nanotechnologies to manipulate and track stem cells, Cell Stem Cell 3 (2008) 136-146.

[51] H. Wallrabe, A. Periasamy, Imaging protein molecules using FRET and FLIM microscopy, Curr. Opin. Biotechnol. 16 (2005) 19-27.

[52] A. Verrecchio, M.W. Germann, B.P. Schick, B. Kung, T. Twardowski, J.D. San Antonio, Design of peptides with high affinities for heparin and endothelial cell proteoglycans, J. Biol. Chem. 275 (2000) 7701-7707.

[53] D. Ding, D. Mao, K. Li, X. Wang, W. Qin, R. Liu, et al., Precise and long-term tracking of adipose-derived stem cells and their regenerative capacity via superb bright and stable organic nanodots, ACS Nano 8 (2014) 12620-12631.

[54] H.H. Wang, C.A.J. Lin, C.H. Lee, Y.C. Lin, Y.M. Tseng, C.L. Hsieh, et al., Fluorescent gold nanoclusters as a biocompatible marker for in vitro and in vivo tracking of endothelial cells, ACS Nano 5 (2011) 4337-4344.

[55] R.H. Lee, A.A. Pulin, M.J. Seo, D.J. Kota, J. Ylostalo, B.L. Larson, et al., Intravenous hMSCs improve myocardial infarction in mice because cells embolized in lung are activated to secrete the anti-inflammatory protein TSG-6, Cell Stem Cell 5 (2009) 54-63.

[56] G. Liu, R.A. Pareta, R. Wu, Y. Shi, X. Zhou, H. Liu, et al., Skeletal myogenic differentiation of urine-derived stem cells and angiogenesis using microbeads loaded with growth factors, Biomaterials 34 (2013) 1311-1326. 